\title{
Case Design for the Data Transmission Instruction of Teaching of SCM
}

\author{
Chen Guangfeng ${ }^{a,}$, Wang Linxiab, Yang Xiangping ${ }^{c}$, Li Huimin ${ }^{\mathrm{d}}$, Li Yan \\ College of Mechanical Engineering, Donghua University Shanghai, 201620, China \\ achengf@dhu.edu.cn, bsnowlxwang@163.com, cyangxp@dhu.edu.cn, ${ }^{\mathrm{d}}$ lihm12@dhu.edu.cn, ${ }^{\mathrm{e}}$ liyanly@dhu.ed \\ u.cn, ${ }^{*}$ corresponding author
}

Keywords: Single chip microcomputer; data transmission instruction; case teaching; software simulation

\begin{abstract}
For the convenience of instruction education of teaching of SCM, multi teaching cases were designed covers all the data transmission instruction, with the help of solutions for teaching demonstration in the simulation software, can vividly display case execution process. Through case teaching demonstration, it can arouse the interest and enthusiasm of the students, effectively promote the students to master the data transmission instruction, and achieve better teaching results.
\end{abstract}

\section{Introduction}

The principle and application of microcomputer is a course with strong theoretical and practical nature. It is a required course for the majority of mechanical engineering, automation, computer and other specialties [1]. This course has much knowledge and abstract content, and the students generally feel that the course is difficult to master. Many educators are exploring ways to raise the teaching effect of this course to improve students' interest in learning, so that students can not only master the basic knowledge of SCM, but also apply them to practical application [2-4].

Teachers try various methods to explore how to make the students fully master the concept and method of the design of the SCM application system [5]. In order to improve the quality of teaching, Proteus simulation software is introduced into the theoretical teaching to directly show the content which is inconvenient to describe in language, and enhance the vividness and intuition of teaching.

The practical results show that the virtual simulation platform based on Proteus and Keil Software is an excellent platform which combines theory and practice for students' study and use [6]. However, due to the limitations of software, the effect of instruction execution cannot be displayed directly in the instruction of assembly instruction.

This paper designs several typical teaching cases based on the Edsim51 software combined with the instruction process of data transmission instruction of 51 single chip.

\section{General Situation of MCS-51 SCM Instruction Teaching}

MCS-51 SCM has 111 assembler instructions. Mastering common instructions is the fundamental of this course. In the course of teaching, how to intuitively demonstrate the effect of instruction and deepen the impression is the content that must be paid attention to. In the teaching process of the assembly instruction of SCM, the instruction is monotonous and boring, the pure text display and explanation are not vivid, it is difficult for students to comprehend and master the related instructions. The existing simulation software such as Proteus has some simulation functions. In the execution, the data variable values are viewed through the debug instruction, but the operation is complex and not very intuitive [7] .

The principle of SCM is used as the first course for electronic majors, which will be used in electronic competitions, technology making, curriculum design and so on. The core goal of the course is to develop the students' hands-on ability [8-9]. SCM instruction runs through the whole course teaching content [10]. The knowledge theory of learning MCU instruction is very abstract. Boring teaching will only enable students to remember some ambiguous instructions mechanically. The application of these instructions is not impressive [11]. 
In the teaching of single chip, we should make full use of the class time, combine with the typical case, and master the instructions skillfully.

\section{General Situation of MCS-51 Data Transmission Instruction}

SCM instruction runs through the whole course teaching content. Master and skilled use of single-chip command, to master the application of the whole chip technology has a very good effect. There are 29 data transmission instructions, which are designed for data transmission, data exchange and stack operation.

Data transmission: MOV, MOVX, MOVC

Stack operation: PUSH, POP

Data exchange: XCH, XCHD, SWAP

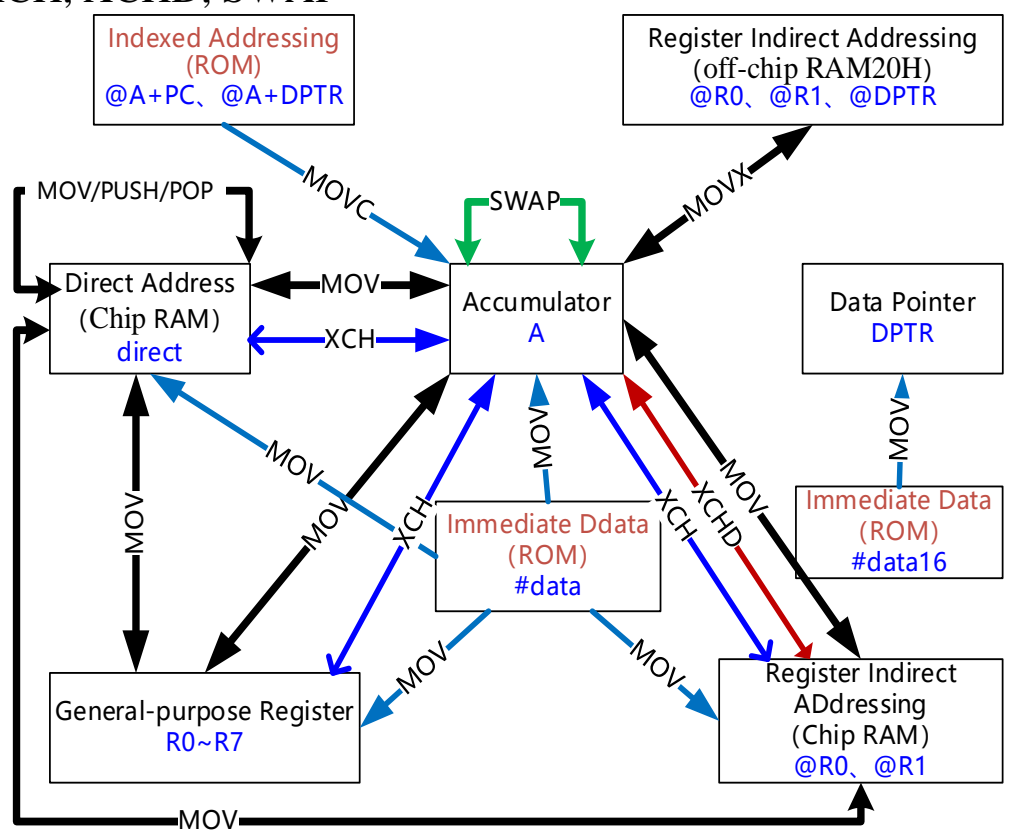

Fig. 1 Diagram of complete data transmission

According to Figure 1, the complete 29 instructions can be written, For example, the instructions that send data to the accumulator Rn can be listed as:

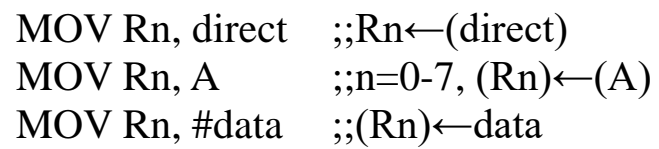

At present, school hours are generally compressed. The time for instruction teaching is also limited. It is difficult to fully master the data transmission instructions and understand the corresponding application in 2-4 class hours. It is necessary to design several typical cases, which cover most of the data transmission knowledge, and demonstrate teaching in the teaching process, which will be beneficial to the instruction of data transfer instruction.

\section{Instruction Case Design of Data Transmission Instructions}

Case Summary Design. As shown in Figure 1, the data transmit instruction involves A, direct, Rn, @Ri, DPTR, @DPTR, \#data, \#data16, @A+PC, @A+DPTR. If all instructions are put into a single case to demonstrate and explanation, the case will be too long, and the difficulty of case design is greatly increased. At the same time, it is easy to cause fatigue in the teaching process.. It can be divided into several typical cases to cover all the instructions, and each case is aimed at a typical application problem. Here are two typical data transmit instruction cases.

Case 1: Data which is taken from ROM $200 \mathrm{H}$ is sent to RAM20H, the $20 \mathrm{H}$ data is sent to $21 \mathrm{H}$, and then send to the register R0 and the off-chip RAM $200 \mathrm{H}$, data from off-chip RAM20H is sent to chip $21 \mathrm{H}$ and R1. (What if the ROM $200 \mathrm{H}$ is changed to $1300 \mathrm{H}$ ? What if R0 is to changed to R7? 
What if off-chip RAM20H is to changed to off-chip RAM200H ? )

Case 2:The 16 bytes in the inner RAM $30 \mathrm{H}$ are written to $0,1,2,3$ and 15 respectively. (If the writing constant is 3 ? If the starting address is 0 , whether it can run normally? How to write if it is written to the off-chip RAM? Can it be achieved if 300 bytes are written continuously?)

Write Case Program. The specific implementation of the case needs to draw a flow chart. According to the flow chart, the register and the RAM space and registers are allocated, the case 2 flowchart is shown in Figure 2.

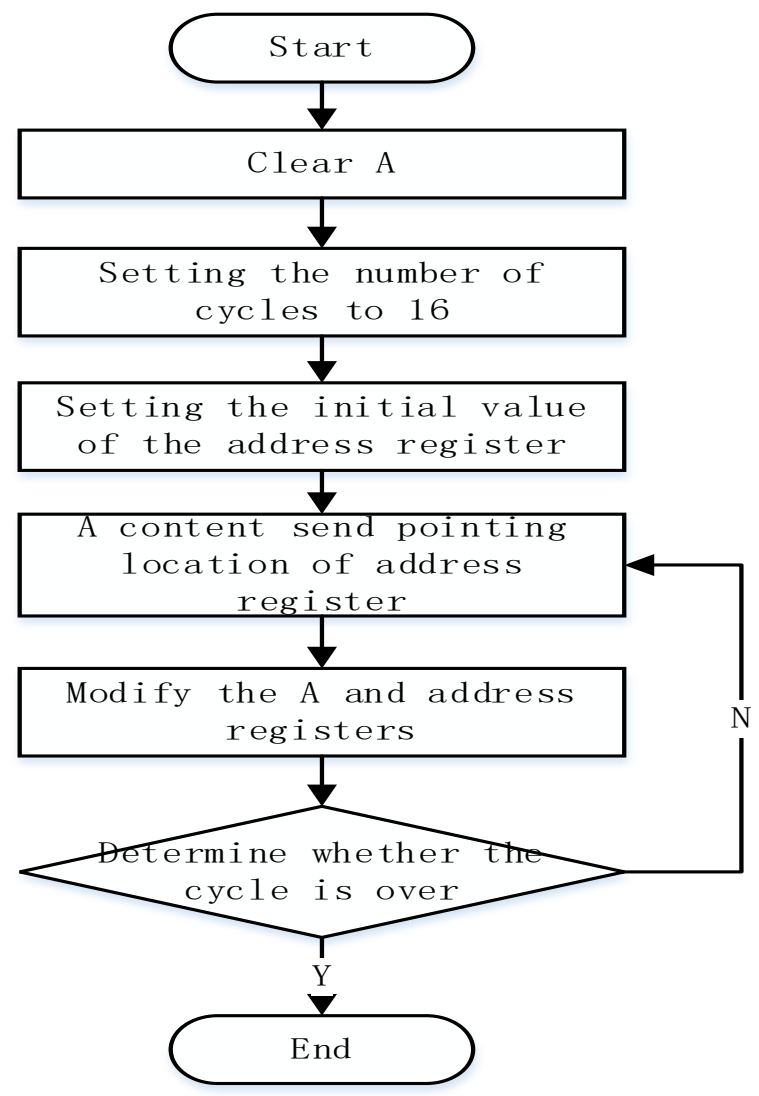

Fig. 2 Flow chart of case 2

The address register select the R0, the loop count uses the R7, and the DJNZ instruction is used to determine whether the cycle is over.

Table 1 Example Program of case 2

\begin{tabular}{|c|c|c|c|}
\hline & ORG & $0000 \mathrm{H}$ & \\
\hline MAIN: & MOV & A, & $\# 00 \mathrm{H}$ \\
\hline & MOV & R0, & $\# 30 \mathrm{H}$ \\
\hline & MOV & R7, & $\# 10 \mathrm{H}$ \\
\hline LOOP: & & & A \\
\hline & MOV & @R0, & \\
\hline & INC & A & LOOP \\
\hline & DJNZ & R7, & \\
\hline & SJMP & \$ & \\
\hline & END & & \\
\hline
\end{tabular}

Cases Simulation and Demonstration. The existing Keil software and Proteus simulation software have a certain simulation function, and can also carry out the data view to display values of RAM, ROM and register during the execution process. However, the operation of data view is complicated and not intuitive. The case is displayed with the Ed51sim software, its general interface can directly input the program, execute, and intuitively display the value of the memory variable. The system clock frequency is $12 \mathrm{MHz}$ and the interface update frequency 1 s are set after the startup software is started. Program is pasted into program code area and click "step" to run one step. All the registers and RAM data are listed on the left side as shown in Figure 3 . In the process of 
execution, register values and RAM data changes can be observed in real time. The specific change examples are compared to the data changes of Figure 4 and Figure 5.

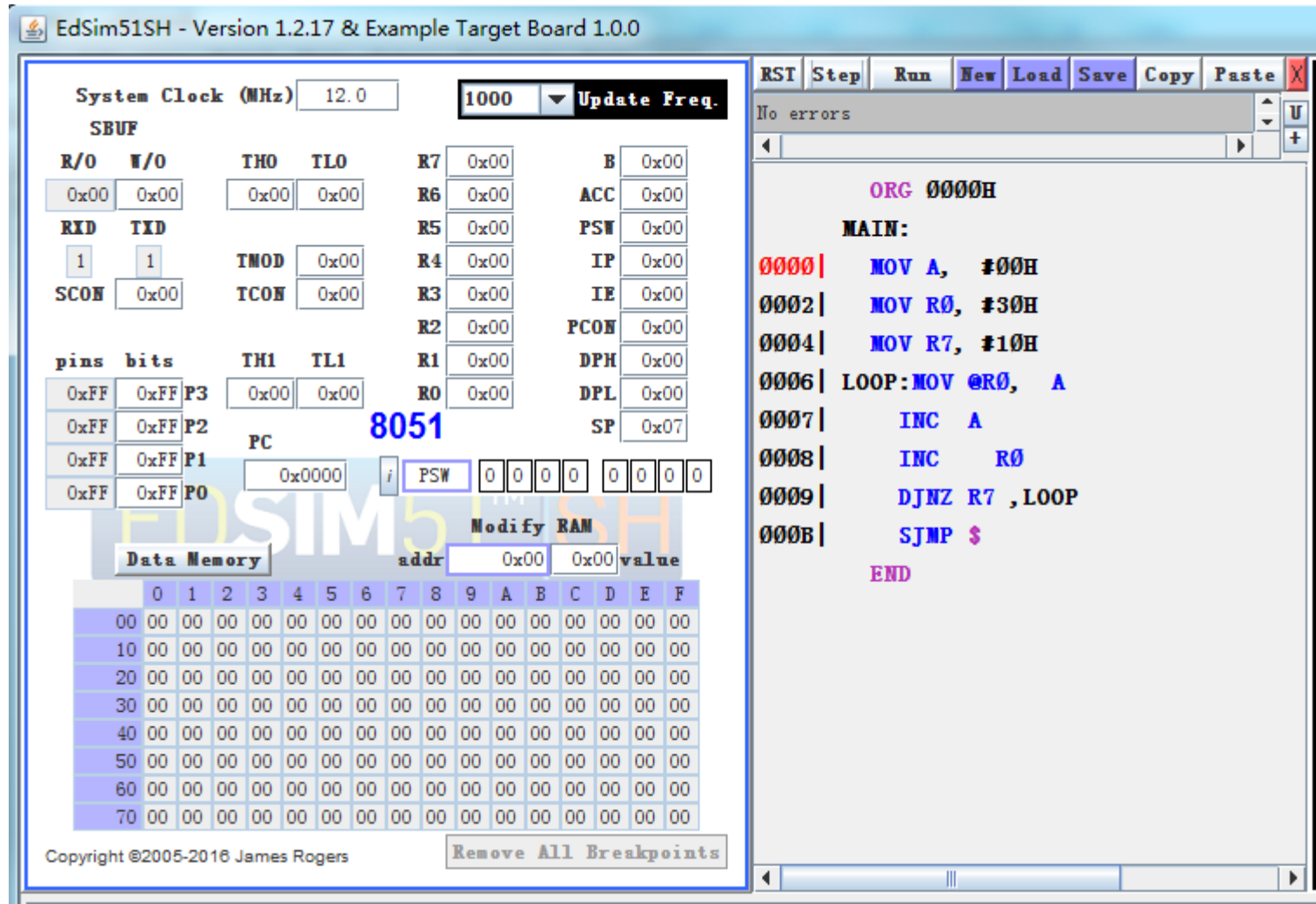

Fig.3 PC=0x0000H before running the simulation

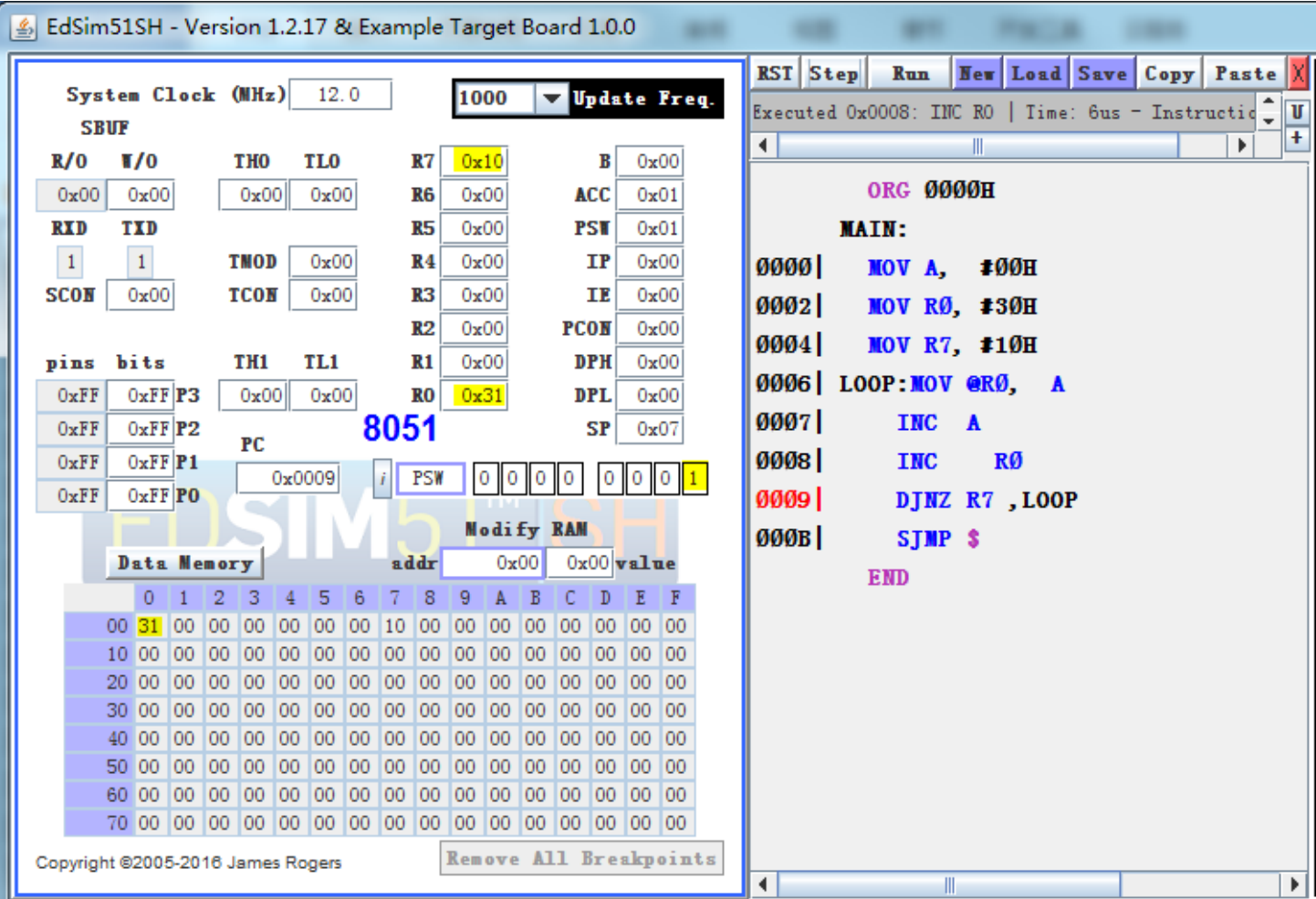

Fig. 4 Simulation effect - state for the first time to execute to the DJNZ instruction 


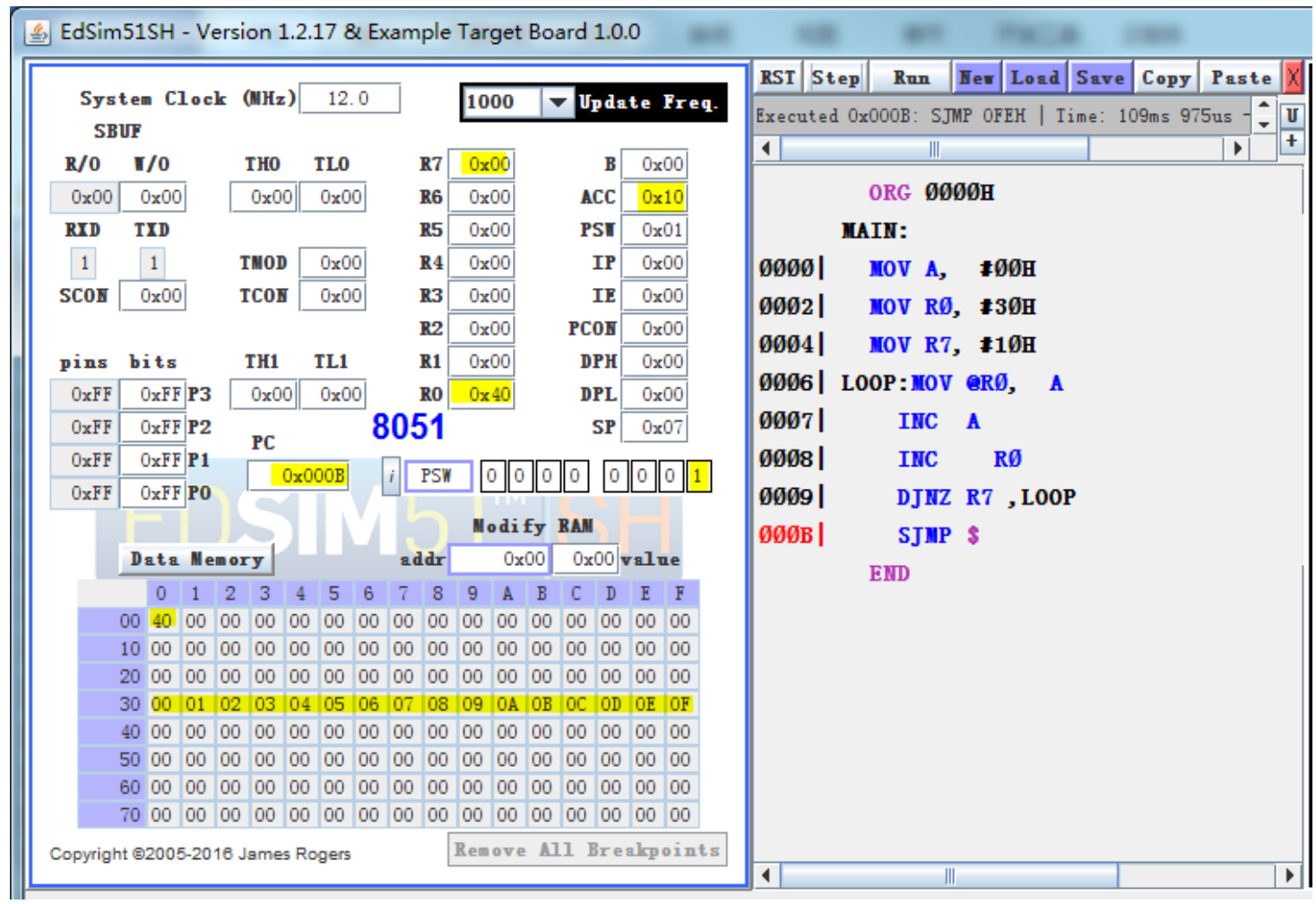

Fig. 5 Simulation effect - Execution end (Marked changed data)

\section{Conclusion}

Through the simulation demonstration, the implementation process of the case program is clear at a glance, which makes the abstract content become image, easy to understand. It is more acceptable than simply learning book knowledge and watching PPT content of teachers, which greatly stimulated students' interest and enthusiasm in learning SCM. At the same time, it can help students to understand and master the case program step by step which will improve the teaching effect. Students can independently design experiments outside class, verify their ideas. It can help train students' innovative quality and creativity, improve the quality of experimental teaching, and expand students' thinking.

\section{Acknowledgments}

I am grateful for the extensive support from the college in the teaching reform of SCM course. At the same time, I should also thank the teachers of the discipline teaching and research group for their precious opinions on the curriculum reform. The teaching reform can be carried out smoothly.

\section{Reference}

[1] Zhang Yigang. Principle and application of SCM. Beijing: Higher Education Press, 2010.

[2] Liu Ming. Analysis of the application of project teaching method in the teaching of single chip microcomputer, J. Course Education Research, 2017, (17): 222-223.

[3] Qiao Jianhua, Li Linsheng, Tian Qichuan. Application of proteus in MCU tTeaching analysis, J. Journal of Electrical and Electronic Engineering Education. 2008. 30 (3): 70-73.

[4] Zhao Yuejing, Chen Jirong, Zhang Yongdi. The innovation and practical teaching reform of the principle and application course of single chip microcomputer, J. Experimental Technology and Management. 2013, 30 (1): 176-179.

[5] Wang Yinghui, Sun Wenfu. Research on the curriculum reform and new teaching model of 
single chip microcomputer, J. Light Industry Science and Technology 2017, 33 (01): 138-139+141.

[6] Wang Yinghe. Research on the teaching reform of SCM course in applied colleges and universities, J. Journal of Higher Education. 2017, (13): 117-119.

[7] Wang Jinggang, Zhang Cuiping. Based on project driven and proteus simulation of MCU teaching reform, J. China Electric Power Education. 2013 (22): 138-139.

[8] Hou Jiatong, Li Quanli, Wang Yulong. The application of proteus in the teaching of SCM course, J. China Modern Educational Equipment. 2015 (10): 69-72.

[9] Xie Qilin. Prospect of practice and thinking of teaching and project teaching combining, J. Microcontroller Technology. 2017, 27 (02): 216.

[10] Tang Yonghong. Explore of singly chip computer experimental teaching,J. JOURNAL OF EEE. 2017,02(29):72-73+81.

[11] Shi Guoying, Li Tianhua, Hou Jialin, Huang Zaifan. Teaching reform on SCM based on proteus and keil, J. Laboratoy science. 2014,17(05):92-94. 